Document downloaded from:

http://hdl.handle.net/10251/47178

This paper must be cited as:

Albero Albero, T.; Sempere Paya, VM.; Mataix Oltra, J. (2013). Analysis and evaluation of the establishment and maintenance of paths in a MANET used for industrial process monitoring. Simulation Modelling Practice and Theory. 37:79-98. doi:10.1016/j.simpat.2013.05.009.

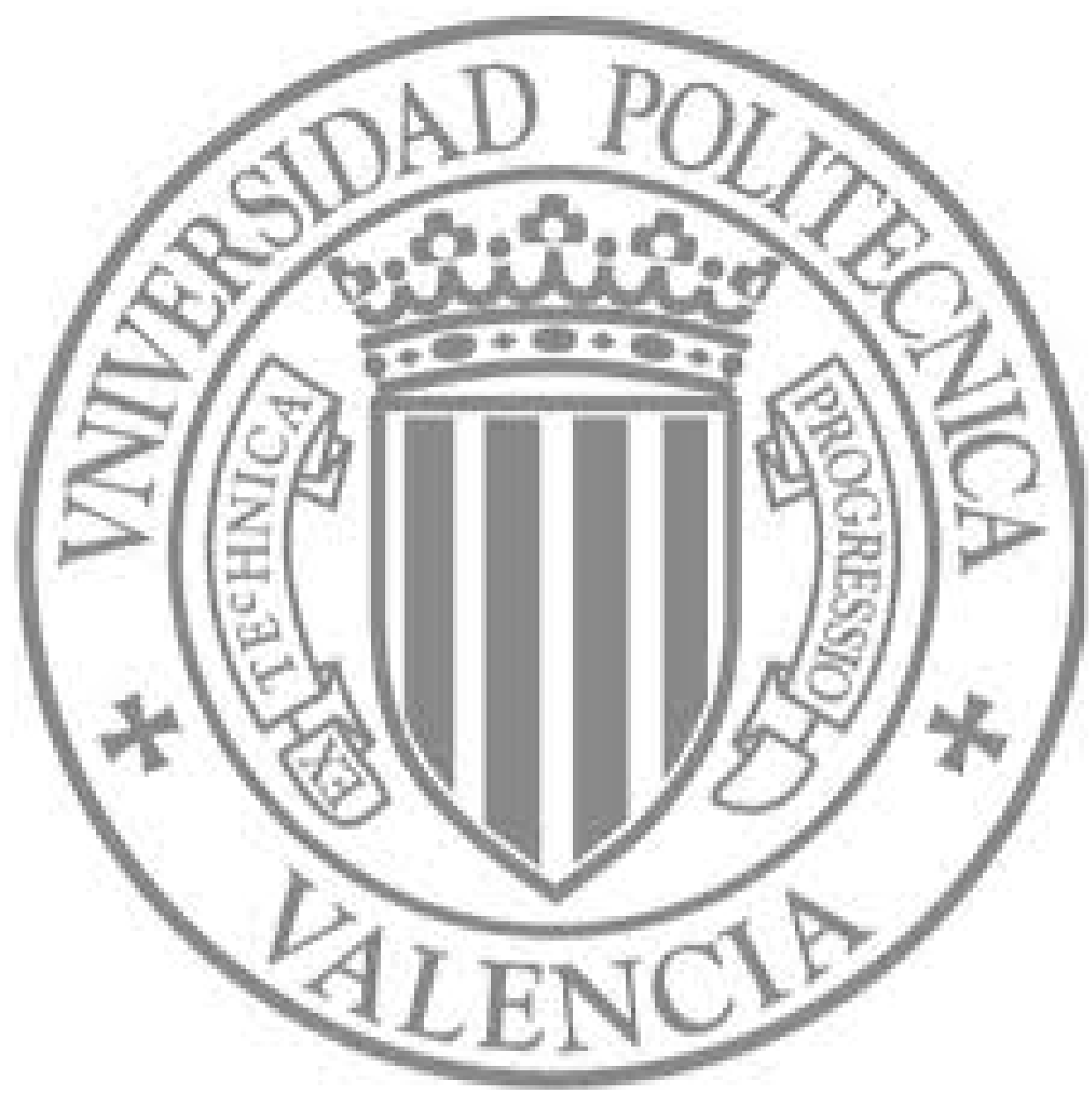

The final publication is available at

http://dx.doi.org/10.1016/j.simpat.2013.05.009

Copyright Elsevier 


\title{
Analysis and evaluation of the establishment and maintenance of paths in a MANET used for industrial process monitoring.
}

\author{
Teresa Albero-Albero ${ }^{a}$, Víctor-M. Sempere-Payáa ${ }^{\text {, Jordi Mataix-Oltra }}{ }^{b}$ \\ ${ }^{a}$ Instituto Universitario Mixto Tecnológico de Informática, \\ Universitat Politècnica de València, \\ Camino de Vera s/n, 46022 Valencia, España \\ ${ }^{b}$ Universitat Politècnica de Catalunya (UPC) \\ Av. del Canal Olímpic, s/n, 08860 Castelldefels, España
}

\begin{abstract}
The use of wireless networks is having an ever greater impact on systems controlling automation, industrial manufacturing, distributed control and supervision. Wireless technologies in particular have penetrated quickly the area of control of public utilities networks, and typical examples of this are remote control and supervision of large water, gas or electricity distribution networks. These types of networks are usually formed of a central station and different remote stations, and remote clients who are able to remotely monitor or even control the working of the system. To improve the use of the system, the remote stations must have the capacity to exchange information between each other and with the central station. The spontaneous generation of wireless networks in remote stations to exchange information between workers is a possible solution. Taking into account the characteristics of this type of monitoring system, the paper has proposed a scenario (number of nodes, area size, mobility) and has modeled, using stochastic activity networks (SAN), the operation of an ad hoc network that uses a reactive routing protocol to determine if the network is able to offer the typical services of these facilities (images or video streaming and alerts).
\end{abstract}

Email addresses: maalal0@dcom.upv.es (Teresa Albero-Albero), vsempere@dcom.upv.es (Víctor-M. Sempere-Payá), jordi.mataix@upc.edu (Jordi Mataix-Oltra)

Preprint submitted to Simulation Modelling Practice and Theory

March 11, 2013 
Keywords: SAN (stochastic activity networks), modeling, MANET, routing protocols, route maintenance, path average lifetime

\section{Introduction}

The use of wireless networks is having an ever greater impact on systems controlling automation, industrial manufacturing, distributed control, supervision, and almost every other type of integrated network system due to the simplicity of setting up and extending the network and low maintenance needs. Moreover, there are many potentially interesting applications that can make use of these wireless networks, as described by Willig in [1], such as coordination between mobile robots or autonomous vehicles and control and monitoring the functioning of machinery and facilities. The characteristics of the ad hoc network studied in this paper are based on those found in a large urban water treatment plant, but it could be the values of another type of facilities such as these to supervise gas or electricity distribution networks.

A good example of wireless technologies having penetrated an area quickly is in the control of public utilities networks, and typical examples of this are remote control and supervision of large water, gas or electricity distribution networks. The traffic on these networks is composed of control information and the transmission of images. These types of networks are spreading in metropolitan environments, and are usually formed of a principal node denominated central station, different remote stations, and remote clients who are able to remotely monitor or even control the working of the system, see $[2,3]$. To improve the use of the system, facilitate maintenance and to improve the response to alerts and potential risk situations, the remote stations must have the capacity to exchange information on states between each other and with the central station. The spontaneous generation of wireless networks in remote stations where each node in the network is represented by a user that exchange information is a solution to the need for fast and timely information exchange.

Routing in wireless networks and in particularly in ad hoc networks is highly changeable, especially when dealing with mobile nodes (MANET Mobile Ad hoc Network), and for this reason, the conventional routing protocols are not appropriate in these environments to provide the mentioned types of traffic. Moreover, the inclusion of wireless networks in industrial environments carries its own particular problems: errors due to interference, 
which make it difficult to create routes within the established times; breaks in active routes due node mobility; noise from surroundings or from the technology used; and difficulties in finding alternative routes after a loss of communication, which may cause problems when establishing communication as well as errors and losses once the communication is active.

Although there has been a great deal of study carried out on ad hoc networks in recent years, there remain many aspects that need to be studied and improved. There are no formal models that take into account a complete network with knowledge of all the topology and where the nodes are mobile. In this paper, we study node accessibility and how this affects their mobility when offering the service requested. With this objective in mind, we have developed a formal model which represents the behavior of a complete ad hoc network in which the routing protocol has reactive behavior. For the implementation of this reactive protocol, some temporal and functional characteristics of the routing protocol AODV (Ad Hoc On-Demand Distance Vector Routing), see [4], have been considered.

Modeling and evaluation of ad hoc communication networks is not a new area of study, in fact there has been a huge amount of work on mobile networks published in recent years. Many studies on MANETs have been based on simulation. The most popular simulation tools for ad hoc networks are OPNET, see an example in [5, 6], ns-2 [7], and Glomosim, see [8]. However, these are not the only tools available to study this type of network. The tool used to construct the formal model presented in the paper is the publicly available tool Möbius 2.3, [9] which supports the specification of Stochastic Activity Networks models, see [10, 11].

There have been various studies on Petri Networks, which have evaluated different aspects of ad hoc networks. Authors such as Murata et al. [12, 13], were the first to model and simulate a MANET with CPN (Colored Petri Net). They modeled in detail the AODV routing protocol. They studied how mobility affects the AODV protocol, but it is important to point out that they did not know the exact topology of the network, they did not know where was located every node in the area. According to Murata, it is possible to model a MANET without information on its exact graphic structure, but in our opinion, this fact makes it impossible to study a break in a route and therefore its recovery. Other authors have used the Fuzzy Petri Net to design an efficient routing algorithm (RRA, Reliable Routing Algorithm) which allows them to find the routing tree from source to destination in an ad hoc network, see [14]. The algorithm used does not need to estimate the 
topology, it only needs to know the neighbors of each node. The objective is to find the most trustworthy route, but without studying what happens after obtaining one of these routes, unlike the paper under consideration where we study the behavior after the path establishment. This work, [14], uses neither the AODV protocol's behavior nor that of a complete ad hoc network, but concentrates only on the RRA algorithm. Another approach to studying an ad hoc network is to not build the network node by node, as according to the authors the size would be too large to obtain an exact numerical solution. In [15], an ad hoc network is presented using Stochastic Petri nets to model the behavior of a node under the work load generated by the entire network. The authors modeled the occupancy of the entry and exit buffers of the nodes in the network, and the rest of the network was modeled compactly. In this work, authors are not representing in detail the behavior of a protocol nor are they considering node mobility.

In the papers cited previously, $[12,13,14,15]$, the behavior of ad hoc networks was modeled without knowing the exact position of the nodes in the network, as the objective was to evaluate whether or not the protocol could establish communication, or to model an algorithm applied to a routing protocol to see if it would improve the possibility of communication. However, without considering the topology of the network, it is not possible to know the exact route to be taken when requesting a communication between source and destination. Therefore it is impossible to know if a later movement of a node will cause a loss of the route, given that it is not known whether the node that has moved belongs or not to the active route. Once again the need for a formal model of a complete ad hoc network where the topology is known and the nodes are mobile is evident. With such a model it would be possible to know the exact route between source and destination and therefore it would also be possible to study what happens when this is active and one or more of the nodes forming it move.

In our previous works the probability of the source reaching the destination in an ad hoc network was studied in function of radio coverage range [16], determining single-hop and multi-hop communications and failed attempts at communication in which the destination was unreachable. Furthermore, the effect of mobility in an ad hoc network on the duration and stability of the routes in order to determine how the services were affected was studied, see [17]. The model shown in the present paper presents a more realistic behavior as it includes error rates more representative of the errors that may occur in an industrial environment when wireless communication is used. AODV 
protocol parameters which intervene in the search for and recovery of routes have been modeled. Furthermore, in this work we have used a more realistic environment taking into account the characteristics of the typical scenarios in which usually appear the networks under evaluation. Therefore, knowing the temporal requirements of industrial networks of metropolitan scope, and knowing how the routing protocol works during transmission of the typical information used in these systems, we can determine whether or not ad hoc mobile networks generated in these environments fulfill these requirements.

Section 2 lists some characteristics and functioning of AODV protocol. In section 3 we give a brief description of the elements of the modeling tool used. The work scenario (environment, work area, call rate, movement rate...) is described in section 4 . In section 5 we explain in detail each of the models which form the model as a whole. In section 6 we present the results obtained on reachability, establishment, recovery and maintenance. Finally, in section 7 , we present our conclusions and propose future lines of research to be carried out.

\section{AODV protocol}

AODV is one of the routing protocols under active development inside the IETF MANET working group. It is an experimental routing protocol that allows dynamic, self initiating and multi-hop routing between the wireless nodes that participate in an ad hoc network. It enables networks with a large number of nodes and different mobility rates, as well as a great variety of levels of traffic. It is characterized by rapid adaptation to dynamic conditions in the links, low processing and memory needs (an important consideration for small devices such as sensor networks), and low network usage, which together mean high scalability and performance. This is in part due to its reactive behavior (a node only initiates a route search to a destination when it needs to transmit something, but does not save routes to all the possible destinations), preserving the medium for high priority traffic, and avoiding large routing tables for nodes that are never going to communicate with each other. Once a route has been obtained, it will not change as long as the communication between the nodes involved is not lost, assuring the use of stable routes for the maximum possible time and continuity of the data flow. AODV uses three types of control messages: Route Request (RREQ), Route Reply (RREP) and Route Error (RERR). 
Although we will not explain the complete operation of the protocol, see [4] for details, we will explain some characteristics that are modeled in the SAN presented in the paper.

If when an RREQ packet is sent a response is not received after NET_TRAVERSAL_TIME milliseconds have passed, the node must try to obtain a route again broadcasting an RREQ packet. This process must be repeated up to a maximum value of RREQ_RETRIES with the maximum TTL allowed. When the time that the source node must wait to receive a RREP after sending an RREQ packet has been calculated, a backoff exponential binary must be used. That is to say, after the first attempt, the waiting time is equal to NET_TRAVERSAL_TIME milliseconds, the waiting time after the second attempt is $2 \times \mathrm{NET}_{-}$TRAVERSAL_TIME milliseconds, and so on up to additional RREQ_RETRIES after the first RREQ.

According to the AODV RFC, if a node moves, although the route is lost in the routing table, this loss does not appear instantaneously, and is not given up as lost until the invalidation time is exceeded. During this time the service can not be visualized by the user because the route is broken, but the protocol still considers the route to be valid. The route is not marked as invalid until the invalidation time has been completed, this time is represented by the value ALLOWED_HELLO_LOSS * HELLO_INTERVAL. By default the protocol allows the loss of 2 HELLO packets (ALLOWED_HELLO_LOSS $=2$ ) to determine if it has lost contact with its neighbor, and the HELLO packets are sent every second (HELLO_INTERVAL $=1000 \mathrm{~ms}$ ). When the route has been invalidated in the routing table the new route search can be initiated.

\section{Modeling tools}

To carry out this study, we used Stochastic Activity Networks, SAN, a stochastic extension of Petri networks to which the possibility to define temporal characteristics with statistical parameters is added. To be precise, we used Möbius ${ }^{T M} 2.3$, see [9], designed by the Performability Engineering Research Group (PERFORM), from Illinois University.

SAN allow us to design networks based on previously defined and evaluated models. As SAN combine graphic design and mathematical theory, it is possible to study the behavior and evolution of the system in a simple way. This allows us to construct the model graphically using basic elements which are interconnected. It is possible to watch the evolution of the model 
over time and this evolution is determined by the conditions imposed in the graphics. We can use mathematical formulas to determine the behavior of the system, as well as theoretical characteristics. To these factors we can also add the possibility of analyzing, executing or simulating by computer a system modeled using SAN. This is the principal characteristic of Möbius, used for the creation of the formal models whose results are presented in this paper.

A SAN has five basic components: places, activities, output gates, input gates, and arcs, see Fig. 1. The activities represent actions in the system modeled which require a certain time interval in order to be completed. There are two types: "timed" (represented by an oval) and "instantaneous" (represented by a bar). With the marking of the places ("tokens"), the state of the system modeled is represented. Each place can house a number of tokens whose meaning is arbitrary and can represent positions, number of requests awaiting service, etc. The places are represented graphically by circles. Möbius has two types of places, a place represented by a blue circle (marked with * in Fig. 1) and a special place denominated "extended place" represented by an orange circle (marked with ** in Fig. 1). The extended places are a new and very powerful tool for modeling complex systems, and can be associated with a structure or an array. The input gates, triangles whose vertices are connected to the activity that they control, are used to control the enabling of activities. The output gates are used to modify the state of the system when an activity is completed. These are represented by triangles, one side of which is connected to an activity or to the case of an activity and its opposite vertex is connected with the exit places by an arc. The arcs connect the different elements in the network, marking their dependence. See more details about formal definitions and basic concepts in $[10,11]$.

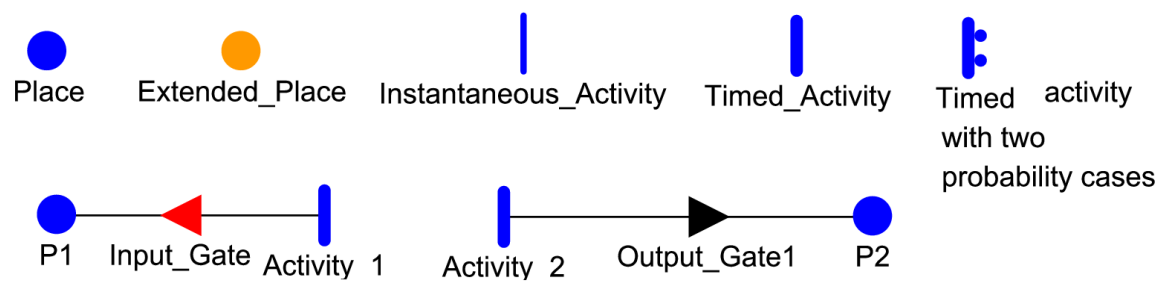

Figure 1: Elements of a Stochastic Activity Network in Möbius 


\section{Scenario}

Knowledge gained from previous work on supervision and control systems, see [18], have enabled us to define in this paper the necessary parameters (radio transmission range, area, number of nodes, movement rate...) which represent a scenario close to reality.

\subsection{Environment}

In this section the type of ad hoc network that is generated, the number of nodes, and the information exchanged that is directly related to the services offered by the system are described.

As mentioned in the introduction, the type of system being studied, a large facilities such as water, gas or electricity networks operating in large metropolitan areas, is generally made up of one central station and various remote stations, see Fig. 2. The central station supervises the system, giving orders or receiving real images from each of the remote stations which give support to the control of the geographically disperse facilities. The remote stations are often large pumping and purification stations. The technical personnel working in each of these often need information on some part of the facilities. So the stations must have the capacity to: a) exchange information on states between them, b) with the central station through a gateway and c) between users within the remote station. In the three cases, one possible scenario is the spontaneous generation of wireless ad hoc networks in the remote stations where each node in the network is represented by a user operator (supervising the states of grilles, relief channels, gates, etc.). This node also acts as a router when information needs to be forwarded to another operator of the same station, the central station or other remote stations. To achieve this, when direct communication is not possible the other nodes in the network are used to relay the information across a new generated path so that the necessary services can be offered. The number of nodes which may constitute the ad hoc network created in a remote control station is related to the number of personnel operating in it, using mobile devices to obtain control or monitoring information. In this type of system, the maximum number of users will be 5 or 6 , and these may be dispersed over an area that will not normally exceed $100000 \mathrm{~m}^{2}$.

In the system used as a scenario, the services offered in real time are those of alerts and media. In the case of alert services, the volume of information is very low, normally a single packet with few octets that give information 


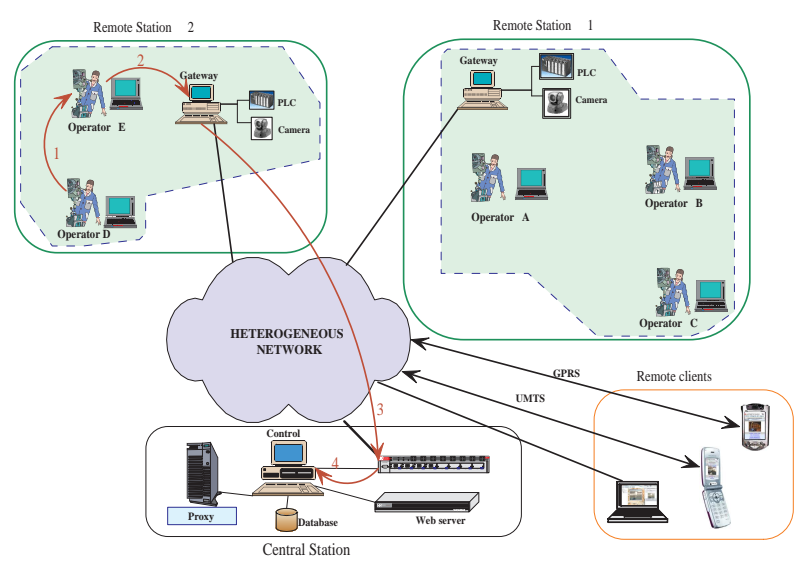

Figure 2: Monitoring and control system of the facilities

on a particular state or alarm in the facilities. The user receives the alarms because he is subscribed to this service. The source of the alarms will always be the control equipment in a remote station. The receiver might be in the central control room, a remote station or may be a remote client. The latency allowed in this case is usually less than $1 \mathrm{~s}$. Media services usually offer images or video streaming to help with supervision of the facilities and the source is usually a camera installed in a remote station. This camera may be fixed or may be remotely controlled. The images or sequences are offered by the camera and sent to a client situated in the station itself, in another remote station, in the central station or even to a remote client. The maximum delay for supervision images or video streaming from the request to visualization by the user is $3 \mathrm{~s}$ or less.

Depending on the service required, a session time is established. This is the time during which the user can observe the information in the screen of his mobile device, whether it is to see the result of a control order given, the state of an alarm received or images needed to supervise a particular part of the facilities. If the information to be transmitted or received is control information or alerts, the session time is usually short (20 s), but if it is necessary to see the images offered by the cameras in order to supervise the working of the facilities, the time used by the user is usually higher $(60 \mathrm{~s}$, 180 s...). Regardless of the session time, the information, images and alerts, is stored on the server for later supervision. 


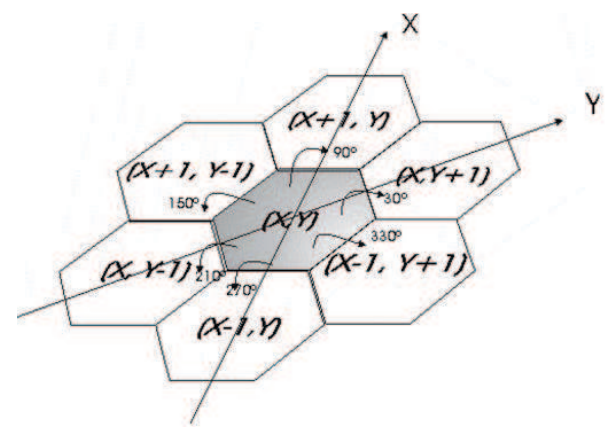

Figure 3: Work area formed with hexagonal cells. Depending on the starting cell and on the angle of movement of the node we obtain the coordinates of the destination cell.

\subsection{Work area}

Another part of the scenario is the work area in which the mobile nodes are to be located. Therefore this section describes the size of the area, how it was divided and numbered in order to know the position of the nodes, and the number of nodes used.

In this case the work area is divided into hexagonal cells. This technique has been used in other works [19],although not for ad hoc networks. In this paper cells are numbered forming what is called a mosaic type $\mathrm{T}$, where the center is a cell. As can be seen in Fig. 3 the cells are numbered beginning with a given cell $(\mathrm{X}, \mathrm{Y})$. The numbering of the cells surrounding $(\mathrm{X}, \mathrm{Y})$ are given depending on the angle of movement when the node pass from cell to cell. The value of the coordinate can be increased or decreased by one unit, that is to say the value can change up $(+1)$ or down $(-1)$, or can be stay without changes. Taking into account that the coordinates of the nodes are represented in the SAN by tokens and that coordinates can not be negative, the coordinates of the central cell must coincide with the size of the mosaic (work area) desired, i.e if the work area is a $T_{3}$ mosaic, the central cell must be $(3,3)$. This work area is shown in Fig. 4 . Whatever the size, the final form of the work area obtained with this numbering is a parallelogram, as can be seen in Fig. 4.

In this area 6 mobile nodes were randomly distributed. A cell can hold more than one node, the position of a node is independent of the rest.

Taking into account the value of the diameter of the hexagon, we can calculate the area in which the mobile nodes are distributed. In Fig. 4 a regular hexagon is shown in which we can see the following elements differentiated: 


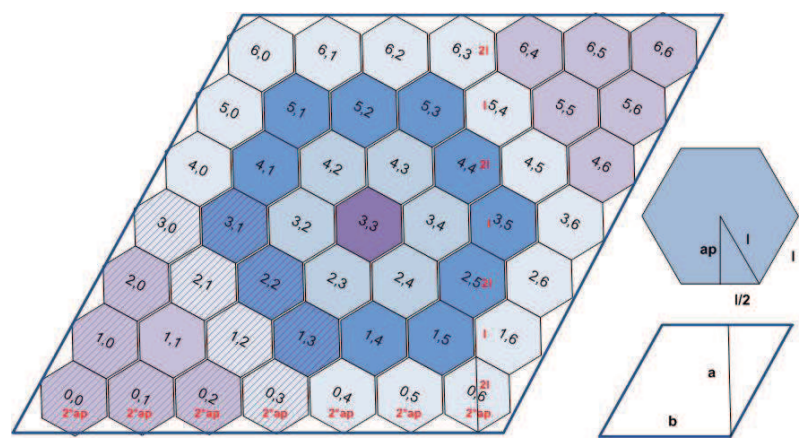

Figure 4: Final shape of the work area made up of hexagonal cells throughout which the mobile nodes are distributed.

ap (apothem), distance from the center of the regular hexagon to the center of one of its sides; and $\mathbf{r}$ (radius), distance from the center of the hexagon to one of its vertices. Considering that in a regular hexagon the side (l) has the same size as the radius, 1 has a value of $25 \mathrm{~m}$, and according the equation 1 the apothem of each hexagon would have a value of:

$$
a p=\sqrt{l^{2}-\left(\frac{l}{2}\right)^{2}}=\sqrt{25^{2}-\left(\frac{25}{2}\right)^{2}}=21.65 m
$$

Therefore, the area for a work zone equivalent to a $T_{3}$ mosaic if the diameters of the hexagons are $50 \mathrm{~m}$ is:

$$
A=b \cdot a=(7 \cdot(2 \cdot a p)) \cdot(11 \cdot l)=303.1 m \times 275 m=83352.5 m^{2}
$$

\subsection{Radio transmission range}

The values of the radio transmission range $(R)$ used are $100 \mathrm{~m}, 150 \mathrm{~m}$ and $200 \mathrm{~m}$. These were nominal ranges, with no variation. The nodes can establish a link when the distance between them is equal to or less than R. As the work area is divided into hexagonal cells and taking into account that each cell has a diameter of $50 \mathrm{~m}$, a node with a radio range of $100 \mathrm{~m}$ can communicate with the nodes situated in the 2 rings around itself. For example, in Fig. 4, we have represented with striped cells the range that a node situated in the cell $(1,1)$ would have if its radio range was $100 \mathrm{~m}$. 


\subsection{Call rate, movement rate and mobility model}

Each time a user requests a service, a route search is initiated to establish the communication. It is related with the communication attempts, these have an exponential time with mean value $1 / \lambda_{c}$, where $\lambda_{c}$ is the call rate.

The length of stay of a mobile node in a cell is characterized by a random variable with a probability density function with the mean value $1 / \lambda_{m}$, where $\lambda_{m}$ is the movement rate. Considering that every cell has a diameter of 50 $\mathrm{m}$, the mean velocity of the users can be calculated with the movement rate. The speed used for the nodes in most of the experiments is $1.38 \mathrm{~m} / \mathrm{s}$, a value equivalent to $5 \mathrm{Km} / \mathrm{h}$, the speed of a person walking normally. However, in some experiments, a higher range of speeds ${ }^{1}$ was used to study the effect of mobility, $5 \mathrm{~m} / \mathrm{s}, 6 \mathrm{~m} / \mathrm{s}, 7 \mathrm{~m} / \mathrm{s}, 8 \mathrm{~m} / \mathrm{s}, 9 \mathrm{~m} / \mathrm{s}, 10 \mathrm{~m} / \mathrm{s}, 15 \mathrm{~m} / \mathrm{s}$, and $20 \mathrm{~m} / \mathrm{s}$.

When one of the 6 nodes moves, it leaves the cell and it can move with the same probability to every one of the neighboring cells $(p=1 / 6)$. It is a simple mobility model based on random direction. Then it is a random walk mobility model (RW) simplified because we does not assign random speed but the same speed to every node in the simulation. RW is a simple mobility model, but is widely studied and used, see [20, 21]

\subsection{Error rate}

The drawback of simulations are simplifications and assumptions introduced. However, it is necessary to make certain suppositions. In this paper, we assume symmetrical links, perfect channel sensing and ideal channel conditions. For example, we have not modeled in detail signal fading, the hidden node problem, and the exposed node problem. Finally, it is important to note that there is no traffic on the network, and so there are always resources available. However, communication problems arising from these phenomena have been represented generically through an error rate introduced into the models. With this error rate we aim to represent the difficulty of establishing communication after a route search.

Table 1 shows the parameters used in the SAN presented in the paper.

\footnotetext{
${ }^{1}$ Some of the speeds are very high for users moving around the work zone, but these have been used in order to see how speed influences the performance of the network.
} 
Table 1: Parameters used in the model

\begin{tabular}{l|c} 
Parameter & Value \\
\hline Number of nodes & 6 \\
Cell size & $50 \mathrm{~m}$ of diameter \\
Workspace size $\left(\mathrm{m}^{2}\right)$ & $300 \times 275$ \\
$\mathrm{R}(\mathrm{m})$ & $100,150,200$ \\
$\lambda_{c}($ calls $/ \mathrm{s})$ & 0.005 \\
$\lambda_{m}($ num. mvts/s) & $0.1,0.12,0.14,0.16,0.18,0.2,0.3,0.4$ \\
Speed (m/s) & $20,60,180$ \\
Session time (s) & $<1$ second \\
Latency (alerts) & $<3$ minute \\
Latency (images) & seconds \\
Time units (t.u.) in the resolution & $6000 \mathrm{t} . \mathrm{u}$.
\end{tabular}

\section{SAN Model}

\subsection{Composed Möbius model}

The composed model is made up of seven sub-models. For reasons of space the details of the model presented in this paper are shown in [22]. The sub-models are interrelated thanks to the common places that they share. This form of state sharing is known as equivalence sharing, since all submodels have the same relationship to the shared state variable. The section composed model in [22] shows the common places (shared state variables) and the models in which they appear (model state variable name). There follows a description of each of these models, which will enable understanding of the functioning of the composed model as a whole. The composed model allows us to calculate the time that the protocol takes to find a route on demand, the average time that the routes are active and the time that the protocol needs to recover a route or find an alternative route when an active route is lost. Using these times and knowledge of the restrictions of the services to be offered, we can determine whether the service can be offered in a satisfactory way to the user and with the required quality.

- "Search AC", shows the communication attempts between nodes. It evaluates whether the communication is possible and whether it is single-hop or multi-hop. This model represents the behavior of a reactive protocol when searching for a route for the first time both from the point of view of time and also the errors that may be produced during communication with a node which, though in terms of coverage 
is reachable, may be influenced by external factors such as noise, the hidden node, interference, etc.

- The "Position" sub-models represent each of the nodes, indicating their initial position in the work area and their new position after moving.

- "If route change" evaluates whether after a movement of a node belonging to the route this movement leads to a break in the link either with its predecessor or successor.

- "Route invalidation" calculates the time that the protocol must wait before invalidating the route that has suffered a break due to movement of a node.

- In the "Alternative route" sub-model, when the route is invalidated, a new route must be found to continue the communication.

- "Delay recovery" calculates the time that the routing protocol must wait to send a new route request packet, RREQ.

- The "Time" sub-model calculates the time that the route remains active and the times that the services requested by a user are in operation.

\subsection{Model "Search $A C "$}

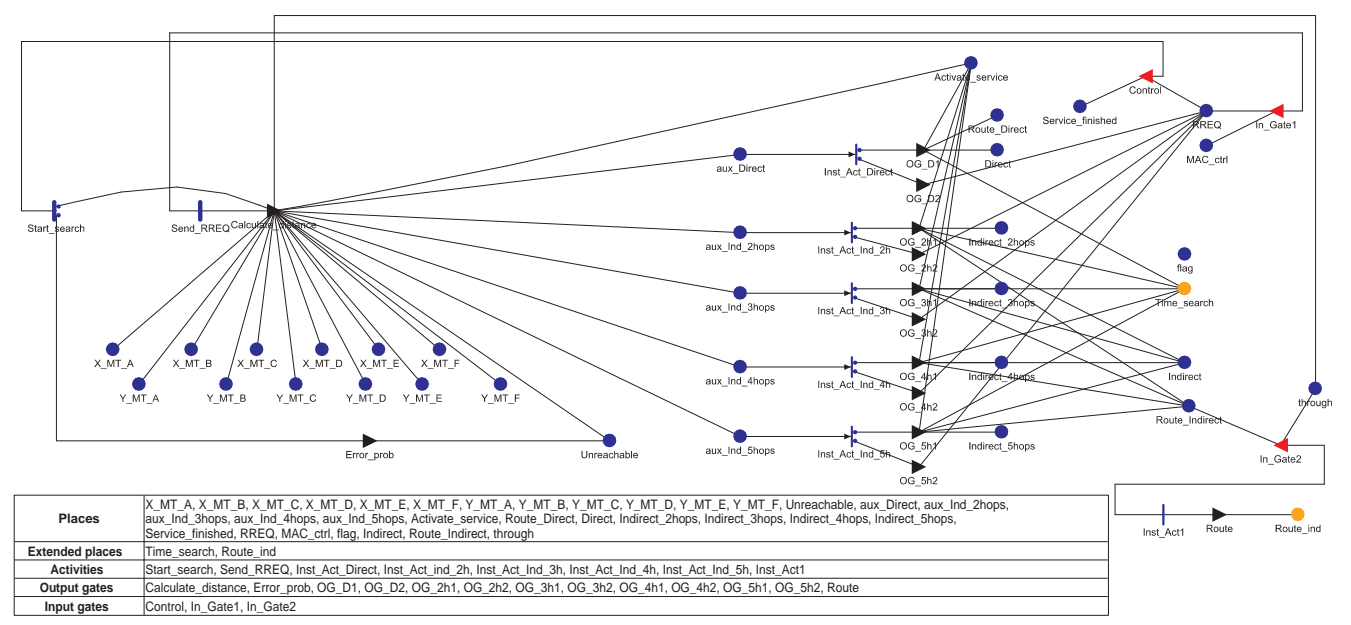

Figure 5: Model "Search AC" 
The search sub-model (see Fig. 5) is used when there is a communication attempt between source - mobile node A, and destination - mobile node C. Note that to simplify the model the source and destination nodes are always the same. The source node sends a route request packet (RREQ) to communicate with the destination. With a call rate of $\lambda_{c}$ the activity "Start_search" is fired. If the first case of the activity is fired, calculation of the route is initiated. Knowing the position of each node, it is possible to determine whether the communication with the destination is single-hop, multi-hop or if the communication is not possible. It is important to remember that we have considered a perfect channel, and that we have not modeled phenomena such as the hidden node, exposed node, etc., but we have represented the problems due to these factors as well as noise by including an error rate which is fired by the second case of the activity "Start_search". In this case the route is given up as unreachable. The same mechanism is modeled in the model "Alternative_route". This error rate gives a little flexibility to the model as, although we are representing fairly generic behavior, it allows us to compare environments with little noise or with visibility problems between nodes with more noisy environments or environments with a lot of interference due to the aforementioned phenomena. It is important to consider that the error rate affects exclusively the route search process.

Going back to route calculation, we want to point out that the exact route is found and stored ${ }^{2}$, that is to say, the nodes participating in the route and the order in which they do so. In the model the first route with least number of hops that is found is chosen. It can be single-hop or multi-hop with a maximum of 5 hops because there are 6 nodes in the network.

Evaluating the behavior of the AODV protocol and studying the results of various real experiments carried out, we know that, although the route is possible in terms of radio coverage, it is not always found at the first request. Here the TTL with which the RREQ packet is sent and the number of hops necessary to reach the destination intervenes. Because of this, in the last part of the model we modeled behavior wherein the probability of a route being considered as definite will depend not only on whether it is possible in terms of radio transmission range but also on the number of attempts carried

\footnotetext{
${ }^{2}$ Discovery and storage of the exact route is costly in terms of modeling. Doing this we can establish whether after the movement of a node in the route, the model is capable of establishing whether this movement affects the route.
} 
out and the number of hops in the route.

For example, in the case that the route is single-hop, independent of the number of attempts represented by tokens in the place "RREQ", the first case of the instantaneous activity "Inst_Act_Direct" is fired and the route is marked as "Direct". This marking is used to count the number of single-hop routes found. Finally the service will be activated (place "Activate_service") because once the protocol has found the desired path the user may enjoy the requested service. A token will also be placed in the corresponding element of the vector represented by the place "Search_times" in order to later know the time intervals in which the routes have been found.

In the case of multi-hop routes, the position of each node in the route is indicated with so many tokens in the corresponding element of the extended place "Route_ind" as their position in the route. This place represents a vector of 4 elements, the first of which is assigned to mobile node $B$, the second to node $D$, the third to node $E$ and the last to $F$. As node $A$ always acts as the source of the route request and node $C$ as the destination, it is not necessary to obtain their position in the route. For example, if the route found was $A-D-B-C$ the mark values in this place will be as follows:

$$
\begin{aligned}
& \text { Route_ind }[0]=2 \Longrightarrow \text { node } B \\
& \text { Route_ind }[1]=1 \Longrightarrow \text { node } D \\
& \text { Route_ind }[2]=0 \Longrightarrow \text { node } E \\
& \text { Route_ind }[3]=0 \Longrightarrow \text { node } F
\end{aligned}
$$

With two tokens in the first element of the vector, it is indicated that node $B$ occupies the second position on the route, with one token in the second element, it is indicated that node $D$ occupies the first position, being this the node that links to node $A$. The nodes with 0 tokens do not take part in the route.

When in this sub-model the service is activated with the place "Activate_service" the time counter is initiated to count the time that the route is active and the service is being offered. This time count is carried out in the sub-model "Time". On the other hand, when the route search gives no result, the search reattempts begin, the value of the place "RREQ" increases and the activity "Send_RREQ" is enabled again.

\subsection{Model "Position"}

This sub-model represents the position of each node in the area and its movement around the area. In the case of node A, marking of places 


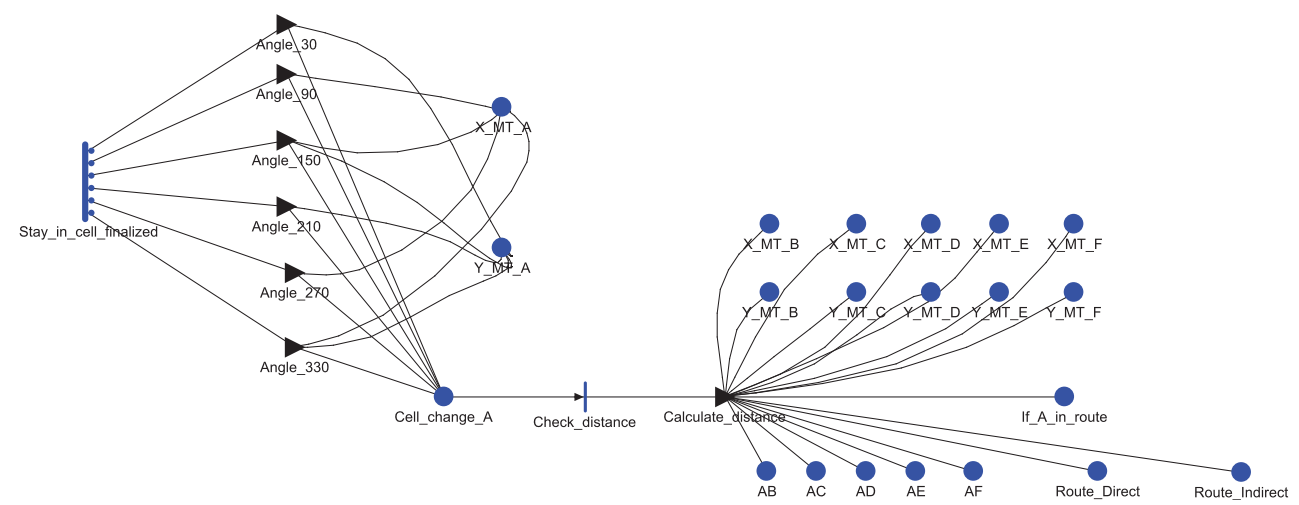

Figure 6: Model "Position MT A"

"X_MT_A" (axis X of the mobile node $A$ ) and "Y_MT_A" (axis Y of the mobile node $A$ ) indicates in which cell of the area the mobile node is located. According to the angle of movement, the marking of these places will vary, indicating the cell in which the node will be located after moving ${ }^{3}$. If the node changes cell, the distance between the node that has moved and the rest must be updated. Distance calculations are carried out in the output gate "Calculate_distance" knowing the position of the rest of the nodes thanks to their coordinates and the current node. The distance is represented with a number of tokens equal to the number of rings separating the nodes, and this marking is located in the corresponding place, "AB", "AC", "AD", "AE" and "AF". Note that this calculation is a "model artifact"; in the real network the distance between the nodes is not known beforehand.

Furthermore, if the node that has changed cell belongs to an active route, it is necessary to evaluate whether this movement means a break in the link between nodes, and therefore a loss of the route, or alternatively whether in spite of this movement the route remains active. This evaluation is performed in the model "If route change".

\subsection{Model "If route change"}

In this model we evaluate whether the movement of a node belonging to an active route has caused a break in the route or not, see Fig. 7. Its functioning is related to the position models, and the places "If_X_in_route"

\footnotetext{
${ }^{3} \mathrm{~A}$ movement does not always mean a change of cell.
} 


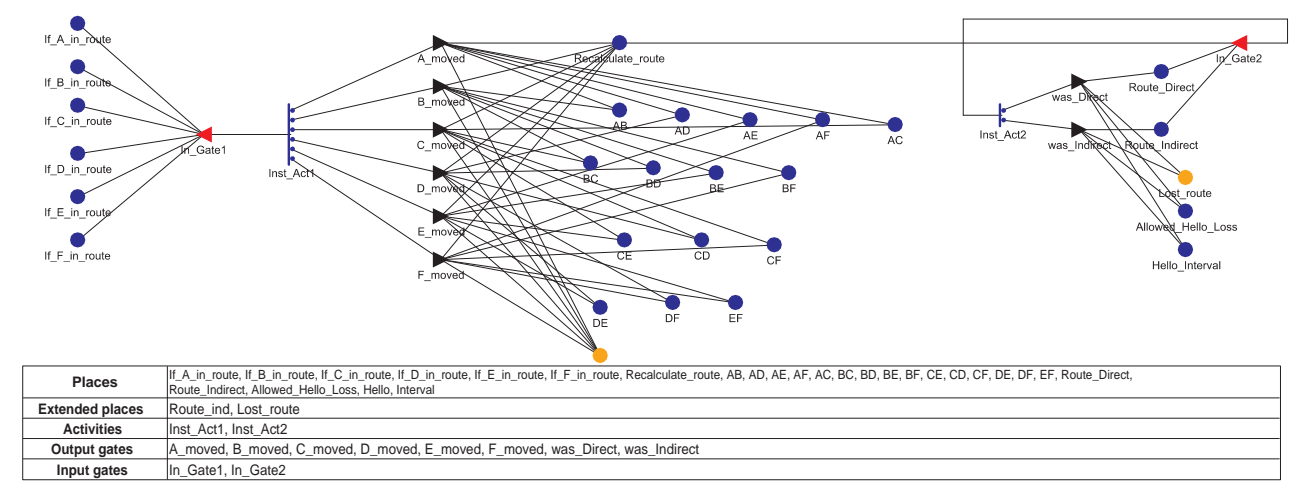

Figure 7: Model "If route change"

being $\mathrm{X}$ whichever of the 6 nodes, will have a token that has been assigned if the node that has moved belongs to the active route ("Position" model).

The current route is obtained by evaluating the extended place "Route_ind". Evaluating its marking we study the position of the node that has moved in the route and therefore which are its predecessors and successors to evaluate only whether the distance between these two nodes is superior to the radio transmission range being used. If it is not, no action is taken and the route continues as before and if any distance is superior to the radio transmission range, it is indicated in the place "Recalculate_route". A token in this place indicates that the route that was active has changed due to the movement of a node. In the output gates "Was_Direct" and "Was_Indirect" the route is given up as lost, depending on whether the route was single-hop or multi-hop respectively, putting the token in the place "Lost_route". This place is shared by the model "Time". Also at this time, the invalidation time is activated, at the end of which the route is marked in the routing table as invalid, an action which allows a new route search to be carried out.

\subsection{Model "Route invalidation"}

Fig. 8 shows this model where the invalidation time is calculated according to the product of ALLOWED_HELLO_LOSS * HELLO_INTERVAL. The product of these two parameters is the rate at which the deterministic activity "Invalidation_time" is fired, an activity which has been enabled when in the model "If route change" a token has been added in the places "Allowed_Hello_Loss" and "Hello_Interval". Therefore, the activity is fired after $2 \mathrm{~s}$ have passed when considering the default values of the AODV protocol. 


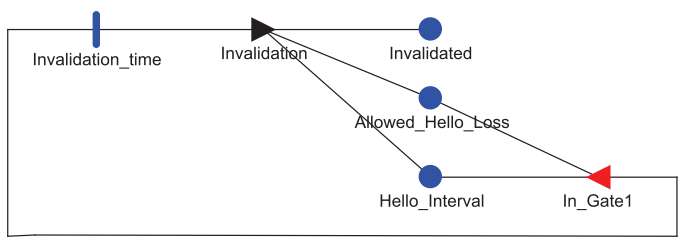

Figure 8: Model "Route invalidation"

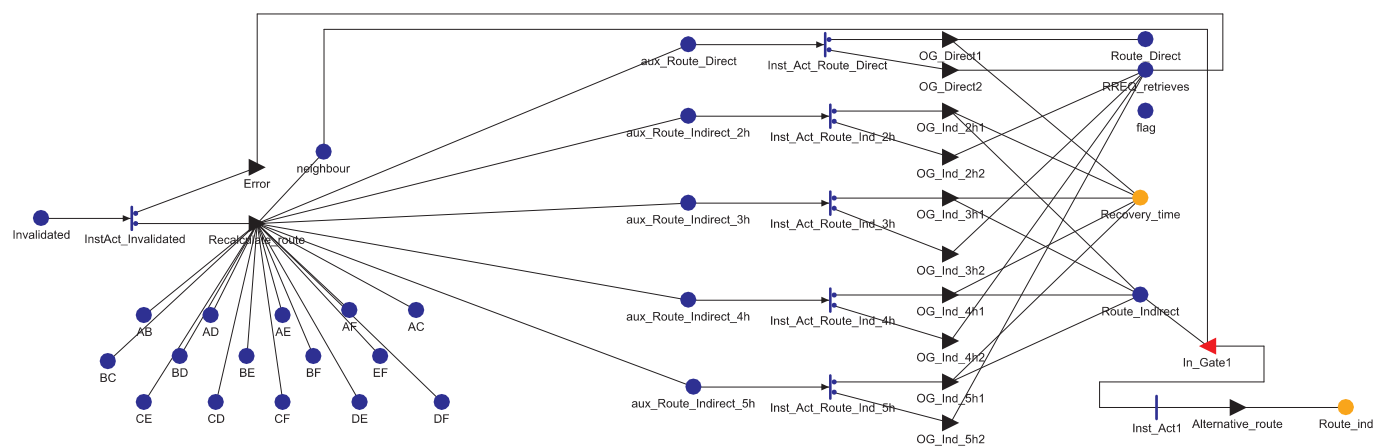

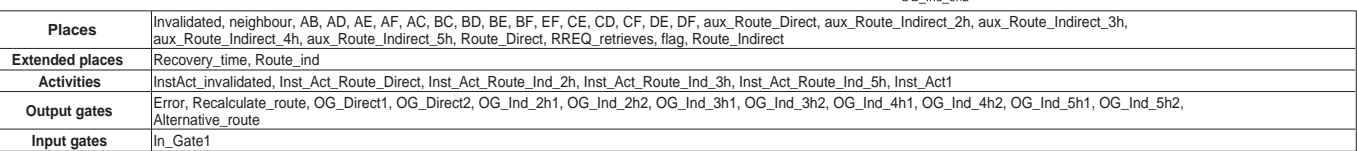

Figure 9: Model "Alternative route"

After this, the values of the places "Allowed_Hello_Loss" and "Hello_Interval" are initiated, and a token is put in the place "Invalidated", a place shared by the model "Alternative route".

\subsection{Model "Alternative Route"}

This model represents the route search for an alternative route to the one being previously used before the loss of the route due to movement of a node, see Fig. 9. The same as occurs in the "Search_AC" model, we modeled an error probability at the moment of the route search, an error which prevents the communication from being established even though there is a possible route in terms of reachability between nodes. On the other hand, if the search for an alternative route is initiated, the calculations are done knowing the distances between nodes with the places "AB", "AC", "AD"..., places shared by the different "Position" models. If a possible route exists, a 


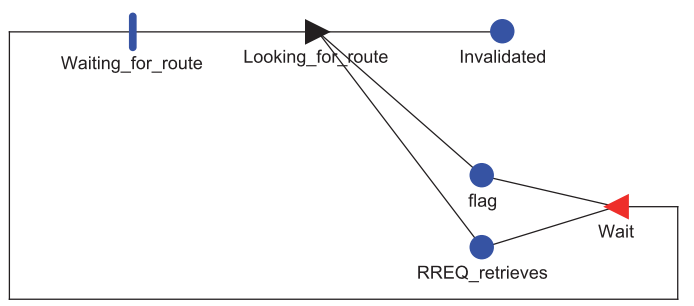

Figure 10: Model "Delay recovery"

token is placed in the corresponding place "aux_Route_Direct" for the singlehop route or whichever of the places "aux_Route_Indirect_Xh", being X the number of hops, if the route is multi-hop.

\subsection{Model "Delay recovery"}

In this model the time that must pass after each route search attempt has been modeled, see Fig. 10. Therefore, when in the model "Alternative route", the alternative route was not found and the number of retries increases, the activity "Waiting_for_route" of this model is enabled. So that, it is fired after the modeled length of time, then the functioning of the "InstAct_Invalidated" of the model "Alternative route" is reactivated.

\subsection{Model "Time"}

This model is used to enable measurement of the average time that the route remains active and to determine the session time, see Fig. 11. Each time a route is found whether it is because the user has requested a service or when the route found is an alternative to a route that has been lost while the service was in operation, a token is placed in "Route_Direct" or "Route_Indirect". It marking fires the activity "t_Direct" or "t_Indirect". The number of times the activity is fired are counted in order to know the number of single-hop or multi-hop routes found during the experiment. Knowing the total time the route is active and the number of routes found (single-hop and multi-hop) we obtain the average time that each route has been active for. The firing of "Route_Direct_lost" and "Route_Indirect_lost" are counted in order to know the number of multi-hop and single-hop routes that have been lost.

We have also modeled the duration which the service remains active. When a route has been found in the model "Search_AC", the service is activated, and the time period during which the user wants to visualize the 


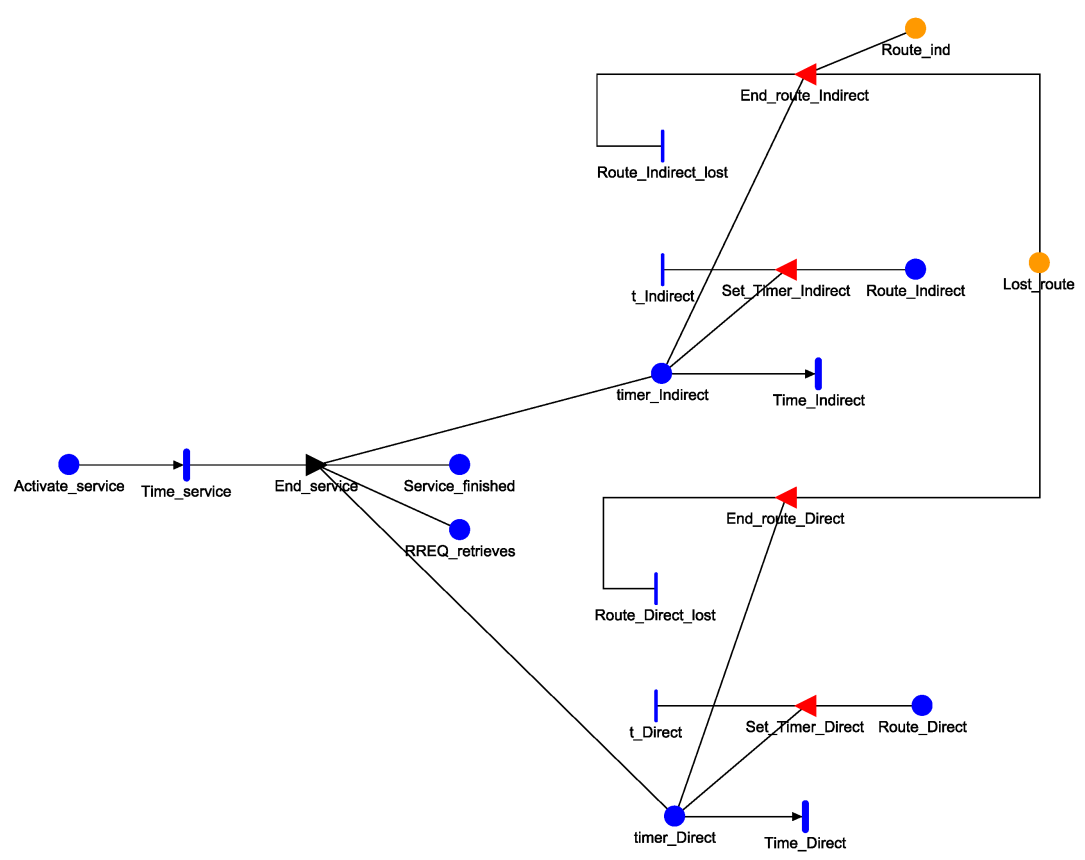

Figure 11: Model "Time"

service begins. It is during this time that we study the behavior of the protocol. The service terminates at the user's request (session time) when the "Time_service" activity has been fired. This time has been given typical values according to the type of service being viewed. When the service is finished, the time that the route is active is no longer counted ${ }^{4}$.

\section{Results}

The results shown were obtained through simulation, numerical analysis is not possible due to the large state space. All of these experiments have been evaluated for a confidence level of 0.95 and a confidence interval of 0.1. The simulation time used was 6000 t.u. (time units).

The results have been divided into three groups: firstly, the results on

\footnotetext{
${ }^{4}$ Although the route remains active when the user ceases to view the information, the model stops counting the route maintenance time because what is really of interest is to know the length of time that the route is active while the service is in use.
} 
reachability of the network when using a scenario such as the one proposed and with a reactive protocol. Secondly, the protocol's behavior in terms of route establishment time, route maintenance time and route recovery time for lost routes. Thirdly, the average time for which the route between source and destination is active.

\subsection{Reachability}

In Fig. 12 single-hop, multi-hop and failed communications are shown, using the node movement speed of $1.38 \mathrm{~m} / \mathrm{s}$ and a value of error rate of $1 \%$. Single-hop routes increase with radio coverage, multi-hop routes show an inflection point for a radio transmission range of $150 \mathrm{~m}$, and failed communications are less when the radio transmission range value used is increased.

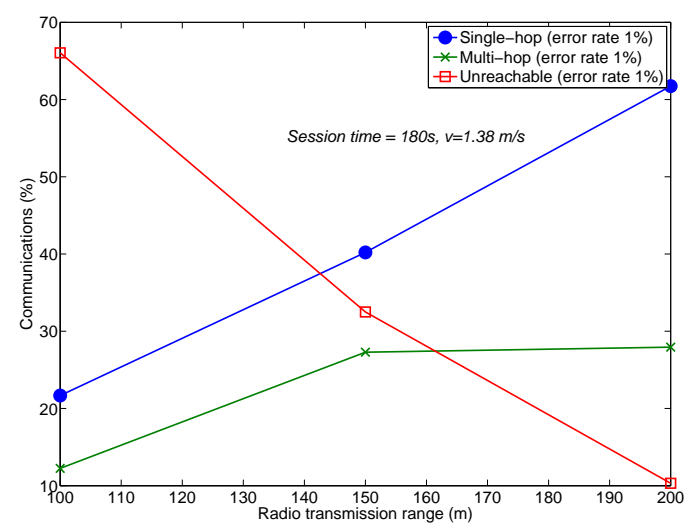

Figure 12: Percentage of single-hop, multi-hop and unreachable communications. Error rate of $1 \%$. Session time $180 \mathrm{~s}$, node speed $1.38 \mathrm{~m} / \mathrm{s}$.

Fig. 13 represents, the percentage of possible single-hop routes, multihop routes and finally communications that were impossible due to node unreachability caused by their position in the area, problems of noise, etc. The results are presented for three radio transmission ranges and different error rates (models have been evaluated for an error rate of 1\%, 10\%,20\%, $30 \%, 40 \%$ and $50 \%$ but here due to space only show the results for three values). Furthermore, their evolution depending on the speed of movement of the nodes is shown. 
In general, we saw for lower error rates that the greater the movement speed the higher the percentage of possible communications as there are more route changes due to node movements. When the error rate increases the slope became flatter and there are hardly any changes with respect to the speed, see Fig. 13(c).

At greater radio transmission ranges there is a higher percentage of singlehop communications ${ }^{5}$. However, in multi-hop communications the network's behavior is different, for example, in Fig. 13(a) for a radio transmission range of $100 \mathrm{~m}$ multi-hop communications reach their minimum, and for a radio transmission range of $150 \mathrm{~m}$ multi-hop communications increase, but curiously, multi-hop communications for a $200 \mathrm{~m}$ range are the same as those with a range of $150 \mathrm{~m}$, or even for some node movement speeds the values are lower than those obtained with this range. To understand this behavior, if we analyze multi-hop communications for a single movement speed and different radio coverage values, we see how at $150 \mathrm{~m}$ this type of communication reaches its maximum value, and thus with $200 \mathrm{~m}$ we see lower percentages (falling values after an inflection point). This behavior with multi-hop communications is seen with whichever error rate, when the radio transmission range is very high as single-hop communications are possible in the majority of communication attempts and multi-hop communication rates fall when using these high radio transmission range values.

Obviously, as the error rate used in the search process increases the protocol has more difficulty in establishing routes between source and destination up to a point where the failed communication percentage is around $75 \%$, see Fig. 13(c).

\subsection{Route establishment and recovery}

In this section the search and recovery times of the routes are evaluated. Firstly, we show the total number of routes found or recovered divided into four graphics according to the route request attempt (RREQ), Fig. 14. Secondly, tables 2, 3, 4 and 5 show the percentage of routes with the multi-hop routes divided according to the number of hops, in order to study in more detail how they are distributed in the time intervals depending on the number that take part in the route.

The routes found after one request and the routes recovered after a break

\footnotetext{
${ }^{5}$ Preference is always given to routes with the lowest number of hops.
} 


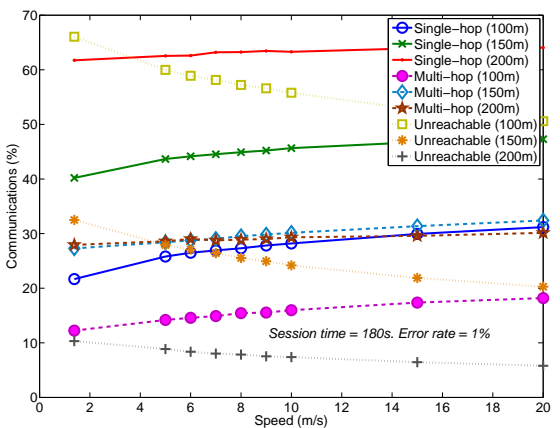

(a) Error rate $1 \%$

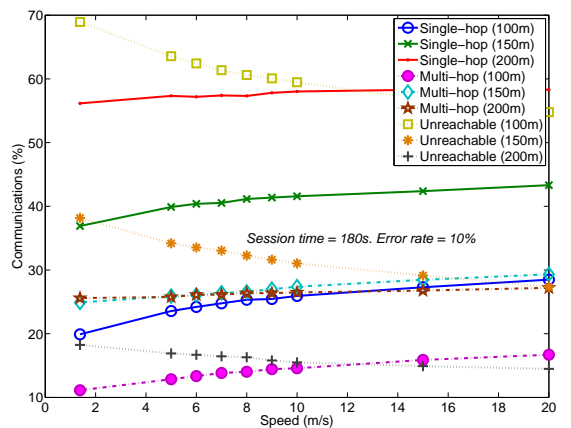

(b) Error rate 10\%

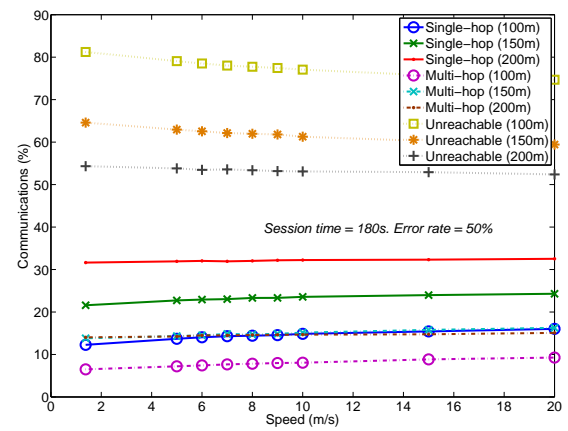

(c) Error rate $50 \%$

Figure 13: Percentages of single-hop, multi-hop and unreachable communications for different radio transmission range values. Session time $180 \mathrm{~s}$, error rates of $1 \%, 10 \%$, and $50 \%$, and different node movement speeds.

have been divided into single-hop and multi-hop. The model does not allow us to know the behavior of the protocol at every instant in time. For this reason we studied it at particular time intervals represented according to the route request attempts. Fig. 14 shows the results classified in four groups: "First RREQ"; "First retry (RREQ)", first attempt to find a new route after a break; and so on successively until the third ${ }^{6}$ and final attempt. These

\footnotetext{
${ }^{6}$ According to the AODV default parameters, there is a first route request and a maximum of two retries (RREQ_RETRIES $=2$ ), and these are the parameters used in the model. The fact that here we use a "Third retry (RREQ)" is another "model artifact", to know whether, after the corresponding waiting time after the second retry, the route
} 
intervals depend on the number of nodes in the network under study and the parameters of the AODV protocol whose characteristics have been used to model the ad hoc network evaluated here. In the case under study, the first time period ${ }^{7}$ is from $0 \mathrm{~s}$ to $0.4 \mathrm{~s}$. The time period evaluated in "First retry RREQ" is between $0.4 \mathrm{~s}$ and $1.2 \mathrm{~s}(0.4 \mathrm{~s}$ plus $0.8 \mathrm{~s}$ which correspond to the waiting time before a new request ${ }^{8}$. If it is necessary to reach a second retry, this would be carried out $1.2 \mathrm{~s}$ after the initial request and from this moment the protocol would wait for the arrival of an RREP packet for $1.6 \mathrm{~s} \mathrm{more}^{9}$, that is $2.8 \mathrm{~s}(0.4 \mathrm{~s}+0.8 \mathrm{~s}+1.6 \mathrm{~s})$ from the initial request. If after this time the route has not been established, the service requested can not be offered given that the number of retries used in the model are those of the default protocol parameters, RREQ_RETRIES $=2$.

In Fig. 14(a), the majority of routes are found, single-hop communications increase with radio coverage, and multi-hop communications have an inflection point. In Fig. 14(b), the majority of routes are multi-hop. This is due to the single-hop routes being found at the first try. If there is no route at the first attempt, and there is also a movement during the waiting time up to making the first retry, it is possible that after the movement there will be a single-hop route, but there will not be many of these routes because the waiting time is not very long and the node movement speed of $1.38 \mathrm{~m} / \mathrm{s}$ does not allow a lot of changes. In the multi-hop routes found after one communication attempt we can still see the inflection point at 150 $m$ radio range. For multi-hop recovered routes the curve diminishes at this point but does reach as far as showing an inflection point. In Fig. 14(c), the behavior is completely different. It is interesting that for both single-hop and multi-hop routes found, and for the routes recovered as single-hop, the graph slope decreases as the radio coverage increases. This is due to the fact that with radio transmission ranges of $150 \mathrm{~m}$ and $200 \mathrm{~m}$ it is easier to find a

\footnotetext{
must be given up as unreachable, and a new route search is necessary, although this is not produced in real experiments. If after this search, there is no route, then this is marked as unreachable or unrecoverable.

${ }^{7}$ In our case NET_TRAVERSAL_TIME $=2 \mathrm{x}$ NODE_TRAVERSAL_TIME $\mathrm{x}$ NET_DIAMETER $=2 \times(40 \mathrm{~ms}) \times 5=400 \mathrm{~ms}=0.4 \mathrm{~s}$

${ }^{8}$ The waiting time for the RREP corresponding to the second RREQ is $2 \times$ NET_TRAVERSAL_TIME milliseconds $=0.8 \mathrm{~s}$.

${ }^{9}$ For each additional attempt, the waiting time for the RREP is multiplied by 2 , then $2 \times 2 \times$ NET_TRAVERSAL_TIME $=1.6 \mathrm{~s}$
} 
route in the previous intervals. After the first try and the first retry, simply due to questions of reachability, given that having high radio transmission ranges, it is not necessary to wait for various attempts before being able to reach the destination whether it be single-hop or multi-hop. In Fig. 14(d) the network's behavior is exactly the same, the slopes are decreasing.

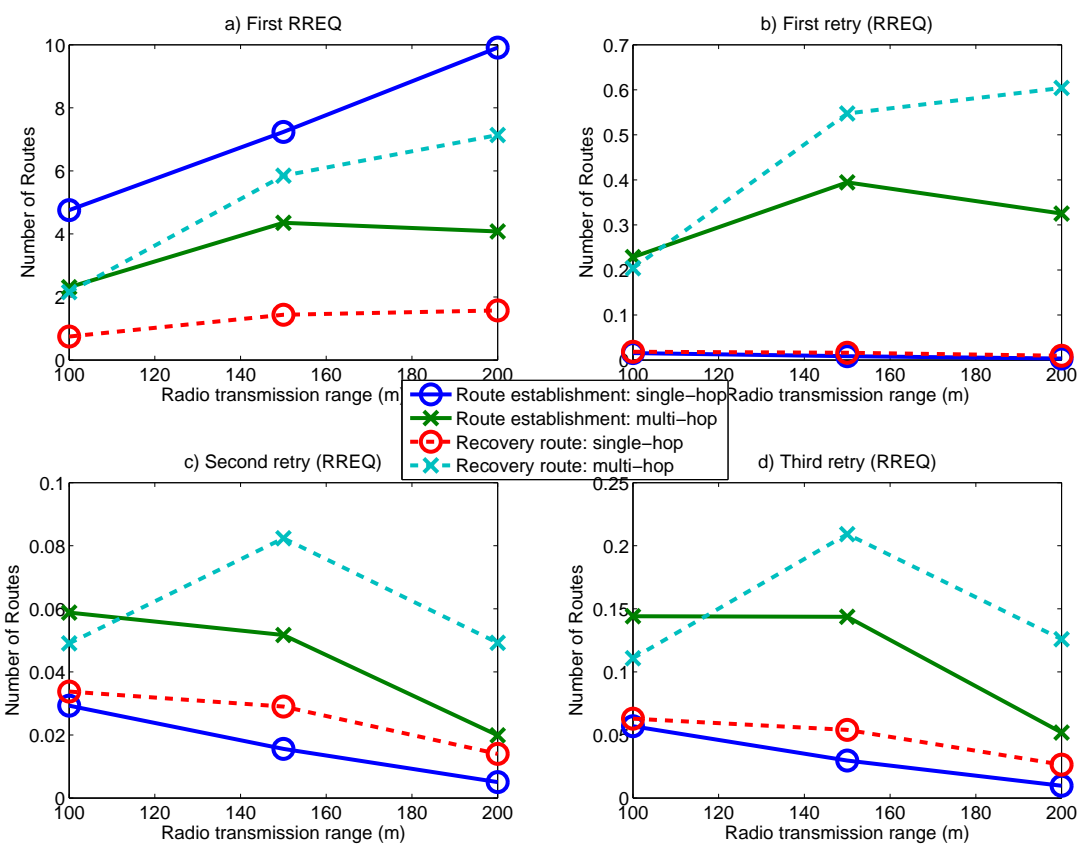

Figure 14: Number of routes found or recovered for each route request packet with an error rate of $1 \%$, an average node movement speed of $1.38 \mathrm{~m} / \mathrm{s}$ and a session time of 180 s. Single-hop and multi-hop routes found according to search attempt.

The total number of routes found or recovered allows us to understand the behavior of the protocol. However it is important to remember that the number of routes established between source and destination depends largely on the number of nodes, the size of the work area, errors during communication, etc. For this reason, in order to evaluate the trends seen in the results for each communication attempt in a fairly general way, the results are calculated with absolute values with respect to the total numbers of routes found or recovered. Tables 2, 3, 4 and 5, show the percentage of routes found or recovered according to the number of hops in the different 
Table 2: Percentage of routes found or recovered after the first RREQ

\begin{tabular}{cccccc} 
Radio coverage $(\mathbf{m})$ & $\mathbf{1}$ hop $(\%)$ & $\mathbf{2}$ hops (\%) & $\mathbf{3}$ hops (\%) & $\mathbf{4}$ hops (\%) & $\mathbf{5}$ hops (\%) \\
\hline Routes found & & & & \\
\hline 100 & 63.1382 & 22.1879 & 6.9532 & 0.8884 & 0 \\
150 & 59.5689 & 26.9123 & 7.4912 & 0.8832 & 0 \\
200 & 68.9328 & 23.6651 & 4.2599 & 0.2853 & 0 \\
\hline Routes recovered & & & & 1.6270 \\
150 & 22.7841 & 51.7941 & 13.8726 & 0 \\
200 & 17.9375 & 54.1005 & 17.7790 & 2.0266 & 0
\end{tabular}

Table 3: Percentage of routes found or recovered after the first retry

\begin{tabular}{cccccc} 
Radio coverage $(\mathbf{m})$ & $\mathbf{1}$ hop (\%) & $\mathbf{2}$ hops (\%) & $\mathbf{3}$ hops (\%) & $\mathbf{4}$ hops (\%) & $\mathbf{5}$ hops (\%) \\
\hline Routes found & & & & \\
\hline 100 & 0.1966 & 1.6316 & 1.1461 & 0.0918 & 0.0747 \\
150 & 0.0670 & 1.8215 & 1.1760 & 0.0894 & 0.0783 \\
200 & 0.0238 & 1.5508 & 0.6520 & 0.0281 & 0.0161 \\
\hline Routes recovered & & & & 0.0646 \\
150 & 0.4719 & 3.0372 & 1.7812 & 0.1285 & 0.0858 \\
200 & 0.1722 & 3.0043 & 2.2198 & 0.1670 & 0.0200
\end{tabular}

intervals of time studied.

The greatest concentration of routes was found or recovered at the first attempt, see table 2 . In the case of routes found, is always given preference to routes with the least number of hops wherever possible. On the other hand, recovered routes are not distributed in the same way. There are more recovered routes of 2 hops than 1 . So if a route is lost, it is unlikely that the recovered route will be single-hop. All single-hop routes that are lost can not be recovered with a single-hop route, and logically, can only be recovered with an multi-hop route. When a route is broken due to the movement of a node, it is more likely that thanks to one or more of the nodes present, it may be reestablished as a multi-hop route.

Routes with 4 hops are very rare and routes with 5 hops are inexistent. There are various reasons that a route might not be found at the first attempt but may be found at the following attempts. One reason is that initially no route was possible, but the movement of a node creates a possible route 
Table 4: Percentage of routes found or recovered after the second retry

\begin{tabular}{cccccc} 
Radio coverage $(\mathbf{m})$ & $\mathbf{1}$ hop (\%) & $\mathbf{2}$ hops (\%) & $\mathbf{3}$ hops (\%) & $\mathbf{4}$ hops (\%) & $\mathbf{5}$ hops (\%) \\
\hline Routes found & & & & \\
\hline 100 & 0.3758 & 0.4496 & 0.2528 & 0.0281 & 0.0187 \\
150 & 0.1185 & 0.2304 & 0.1446 & 0.0266 & 0.0204 \\
200 & 0.0341 & 0.0788 & 0.0445 & 0.0079 & 0.0038 \\
\hline Routes recovered & & & & 0.0303 & 0.0128 \\
150 & 0.7010 & 0.6679 & 0.2390 & 0.0369 & 0.0165 \\
200 & 0.2333 & 0.3914 & 0.1838 & 0.0195 & 0.0040
\end{tabular}

Table 5: Percentage of routes found or recovered after the third retry

\begin{tabular}{cccccc} 
Radio coverage $(\mathbf{m})$ & $\mathbf{1}$ hop (\%) & $\mathbf{2}$ hops (\%) & $\mathbf{3}$ hops (\%) & $\mathbf{4}$ hops (\%) & $\mathbf{5}$ hops (\%) \\
\hline Routes found & & & & & \\
\hline 100 & 0.7342 & 0.8552 & 0.4852 & 0.4378 & 0.0541 \\
150 & 0.2287 & 0.4441 & 0.2717 & 0.3744 & 0.0529 \\
200 & 0.0653 & 0.1514 & 0.0829 & 0.1081 & 0.0092 \\
\hline Routes recovered & & & & 0.0277 \\
150 & 1.0568 & 0.9920 & 0.3679 & 0.3436 & 0.0320 \\
200 & 0.3554 & 0.5937 & 0.2738 & 0.3906 & 0.0079
\end{tabular}

which is found with one of the consequent retries. Another reason could be that there might be interference or noise etc. during the initial route search that make it difficult to establish a route but after the consequent retries a route is found. Finally the initial route found could have 5 or more hops and according to the protocol the first RREQ message is generated with a value of 2 in the TTL field in the IP header. For this it is impossible to find a route with this number of hops at the first attempt, but it is possible at the first or second retry, because the second RREQ is generated with the same parameters but with an increased TTL $=4$.

With the results of the first retry, table 3 , we can draw the following conclusions. They only constitute about $3.5 \%$ of the total route found or recovered. The majority are now of 2 and 3 hops, which is logical given that single-hop routes are found with greater probability at the first attempt. There are some routes of 5 hops but the values are extremely low (less than $0.1 \%$ ). 
Table 6: Average link lifetime for an error rate of 1\%, variable radio coverage from $100 \mathrm{~m}$ to $200 \mathrm{~m}$ and a session time of $20 \mathrm{~s}, 60 \mathrm{~s}$ and $180 \mathrm{~s}$.

\begin{tabular}{cccccc} 
Radio txon range $(\mathbf{m})$ & $\mathbf{1 . 3 8} \mathbf{~ m} / \mathbf{s}$ & $\mathbf{5} \mathbf{~ m} / \mathbf{s}$ & $\mathbf{1 0} \mathbf{~ m} / \mathbf{s}$ & $\mathbf{1 5} \mathbf{~ m} / \mathbf{s}$ & $\mathbf{2 0} \mathbf{~ m} / \mathbf{s}$ \\
\hline Session time $20 \mathrm{~s}$ & & & & & \\
\hline 100 & $14.39 \mathrm{~s}$ & $9.40 \mathrm{~s}$ & $6.58 \mathrm{~s}$ & $5.08 \mathrm{~s}$ & $4.18 \mathrm{~s}$ \\
150 & $15.68 \mathrm{~s}$ & $11.25 \mathrm{~s}$ & $8.34 \mathrm{~s}$ & $6.69 \mathrm{~s}$ & $5.61 \mathrm{~s}$ \\
200 & $17.25 \mathrm{~s}$ & $13.73 \mathrm{~s}$ & $11.02 \mathrm{~s}$ & $9.25 \mathrm{~s}$ & $8.03 \mathrm{~s}$ \\
\hline Session time $60 \mathrm{~s}$ & & & & \\
\hline 100 & $31.75 \mathrm{~s}$ & $15.48 \mathrm{~s}$ & $9.15 \mathrm{~s}$ & $6.58 \mathrm{~s}$ & $5.16 \mathrm{~s}$ \\
150 & $37.08 \mathrm{~s}$ & $20.47 \mathrm{~s}$ & $12.80 \mathrm{~s}$ & $9.33 \mathrm{~s}$ & $7.43 \mathrm{~s}$ \\
200 & $44.36 \mathrm{~s}$ & $28.30 \mathrm{~s}$ & $19.07 \mathrm{~s}$ & $14.43 \mathrm{~s}$ & $11.71 \mathrm{~s}$ \\
\hline Session time $180 \mathrm{~s}$ & & & & & \\
\hline 100 & $51.34 \mathrm{~s}$ & $19.65 \mathrm{~s}$ & $10.64 \mathrm{~s}$ & $7.30 \mathrm{~s}$ & $5.59 \mathrm{~s}$ \\
150 & $64.59 \mathrm{~s}$ & $28.40 \mathrm{~s}$ & $15.70 \mathrm{~s}$ & $10.87 \mathrm{~s}$ & $8.38 \mathrm{~s}$ \\
200 & $82.68 \mathrm{~s}$ & $42.81 \mathrm{~s}$ & $25.22 \mathrm{~s}$ & $17.80 \mathrm{~s}$ & $13.84 \mathrm{~s}$
\end{tabular}

From the second retry, table 4, the percentage of recovered routes again is superior to that of routes found. When routes are recovered, they are mainly of 1 or 2 hops, and these routes have been recovered because during the waiting time a node has moved creating a possible route.

In table 5, the 1 and 2 hop routes recovered dominate the route totals. When there has been a break in a route, there were cases where the route could not be recovered until all this time had passed (3 retries), and at this moment an alternative route was found due to a movement of a node or nodes, and 1 or 2 hops was enough to find a route in the majority of cases.

\subsection{Average link lifetime}

The average link lifetime is the total time (the sum of the parts if there are breaks) at the end of the experiment during which there is a possible route between source and destination, divided by the total number of routes. Table 6 shows the average link lifetime using an error rate of $1 \%$ for a session time of $20 \mathrm{~s}, 1$ and 3 minutes. We can see, how it is logical, that with greater radio range, the time that the route remains active is greater. Moreover, with lower node movement speeds the average link lifetime is greater as the routes are affected less than with higher node movement speeds. 


\subsection{Summary results}

In this work, we have studied how the speed of a node located in an ad hoc network affects reachability, incorporating also in the study an error rate; errors that are produced during the route search and therefore make it difficult to establish communication between source and destination although the route exists theoretically in terms of reachability. As the error rate is increased, communications (single-hop and multi-hop) are affected, resulting in a significant increase in the percentage of failed communications. When the error rates are high, the situation is dominated by failed communications, both single-hop and multi-hop with little difference between them, and independently of the radio coverage used the percentage of satisfactory communications is below $50 \%$.

We have evaluated the route recovery in the case of a rupture. Taking into account the efforts made to search for the path and therefore the time that this represents, we can say that at the first attempt, either for the establishment or for the recovery of a route, are found most of the routes. This is due to two reasons: routes of 1 hop are found (if they exist) always at the first attempt, and the routes of 2 or 3 hops are mostly found at this first attempt. From this data, we can deduce that in the network under study the majority of routes found or recovered take place within 0'4 s, and this means that alert services as well as media services fulfill the defined time requirements from the moment of request, $1 \mathrm{~s}$ and $3 \mathrm{~s}$ respectively, in the great majority of cases when a route is requested. In summary, we can say that in the case of routes with few hops, the required service is established or recovered within the time limit. A delay is observed in establishing routes in topologies with 4 or more hops.

In terms of the average link lifetime, when there is low mobility (1.38 $\mathrm{m} / \mathrm{s}$ ) and the scenario is not very noisy (ER 1\%), for a session time of $20 \mathrm{~s}$ the route is stable between $70 \%$ and $85 \%$ of the time. In the case of images, for a session time of $60 \mathrm{~s}$ the route is stable between $53 \%$ and $75 \%$ of the time. And finally, for a session time of $180 \mathrm{~s}$ the route remains active between $30 \%$ and $50 \%$ approximately of the during which the media service is being viewed. When mobility is greater, the time for which the route is stable is lower. Therefore, we can say that with the default values for the AODV protocol it is possible to offer satisfactory alert services, but media services would be of poor quality. 


\section{Conclusions and future work}

The model has allowed us to identify whether can be offered the typical services of a system as tested. These services are alerts and images or video streaming. Overall, the user will perceive correctly and just in time an alert sent through the ad hoc networks formed in large facilities for industrial process monitoring. On the other hand, the quality with which the user will perceive a service such as images or video streaming will be poor when the default parameters of the AODV are used. This is due to the continuous cuts produced during display.

If a model of an ad hoc network allows us to evaluate its behavior, obtaining results just by modifying the values of parameters or variables, as well as evaluating different aspects by simply implementing new models, then it is certainly necessary to compare and contrast these results obtained via simulation with real experiments. With this in mind, the results presented in this paper could be compared to those obtained from a real testbed created to test the working of the AODV routing protocol in an ad hoc network, [23]. The protocol performed at a high level in the tests, showing the capacity to find routes almost instantaneously as in the model and showing cuts in the video streaming transmission with the default parameters of the AODV protocol.

\section{Acknowledgment}

This work was supported by the MCyT (Spanish Ministry of Science and Innovation) under the projects TIN2010-21378-C02-02, whose are partially funded by FEDER. The first author has been further supported by the ITI (Instituto Tecnológico de Informática).

\section{References}

[1] A. Willig, Recent and emerging topics in wireless industrial communications: A selection, IEEE Transactions on Industrial Informatics 4 (2008) 102-124.

[2] V. Sempere, T. Albero, J. Silvestre, Remote access to images and control information of a supervision system through gprs, in: Proceedings of 1st IFAC Symposium on Telematics Applications on Automation and Robotics, 2004. TA’04, Helsinki, Finland, pp. 107-112. 
[3] T. Albero, V. Sempere, J. Silvestre, P. Dabbas, Environmental control system based on mobile devices, in: Emerging Technologies and Factory Automation, 2005. ETFA 2005. 10th IEEE Conference on, volume 1, pp. $8-838$.

[4] C. Perkins, E. Royer, Ad-hoc on-demand distance vector routing (aodv), rfc 3561, http://www.ietf.org/rfc/rfc3561.txt.

[5] F. Alnajjar, Snr/rp aware routing algorithm: Cross-layer design for manets, International journal of wireless and mobile networks (2009).

[6] J. Y. Hou, Mobile ad hoc network of simulation framework based on opnet, in: Computer, Mechatronics, Control and Electronic Engineering (CMCE), 2010 International Conference on, volume 1, pp. $132-135$.

[7] S. Mohapatra, P. Kanungo, Comparative performance analysis of manet routing protocols using ns2 simulator, in: V. V. Das, N. Thankachan (Eds.), Computational Intelligence and Information Technology, volume 250 of Communications in Computer and Information Science, Springer Berlin Heidelberg, 2011, pp. 731-736.

[8] M. Bhardwaj, S. Pandey, R. Mahapatra, Problem analysis of routing protocols in manet in constrained situation, International Journal on Computer Science and Engineering (2011) 2748.

[9] MöbiusTM. Model-Based Environment for Validation of System Reliability, Availability, Security, and Performance. User Manual, version 2.3.1. May 5, 2010, Performability Engineering Research Group (PERFORM).

[10] J. F. Meyer, A. Movaghar, W. H. Sanders, Stochastic activity networks: Structure, behavior, and application, in: International Workshop on Timed Petri Nets, 1985, Turin, Italy, pp. 106-115.

[11] W. H. Sanders, J. F. Meyer, Stochastic activity networks: formal definitions and concepts, Springer Lectures On Formal Methods And Performance Analysis, first EEF/Euro summer school on trends in Computer Science (2002) 315-343.

[12] C. Xiong, T. Murata, J. Tsai, Modeling and simulation of routing protocol for mobile ad hoc networks using colored petri nets, in: Proceedings 
of the conference on Application and theory of petri nets: formal methods in software engineering and defence systems, 2002, volume 12 of CRPIT'02.

[13] C. Xiong, T. Murata, J. Leigh, An approach for verifying routing protocols in mobile ad hoc networks using petri nets, in: Emerging Technologies: Frontiers of Mobile and Wireless Communication, 2004. Proceedings of the IEEE 6th Circuits and Systems Symposium on, volume 2, pp. $537-540$.

[14] H. Ma, Z. Hu, G. Wang, A reliable routing algorithm in mobile ad hoc networks using fuzzy petri net, in: Global Telecommunications Conference Workshops, 2004. GlobeCom Workshops 2004. IEEE, pp. 80 -84 .

[15] C. Zhang, M. Zhou, A stochastic petri net-approach to modeling and analysis of ad hoc network, in: Information Technology: Research and Education, 2003. Proceedings. ITRE2003. International Conference on, pp. $152-156$.

[16] T. Albero, V. Sempere, J. Mataix, A study of mobility and reachability in ad hoc networks using stochastic activity networks, in: Next Generation Internet Design and Engineering, 2006. NGI'06. 2nd Conference on, pp. $86-93$.

[17] T. Albero-Albero, V.-M. Sempere-Payá, J. Mataix-Oltra, Study of the path average lifetime in ad hoc networks using stochastic activity networks, Lecture Notes in Computer Science, Springer Proceedigs of the 16th International Conference on Analytical and Stochastic Modelling Techniques and Applications, ASMTA'09 5513 (2009) 71-88.

[18] V. Sempere, T. Albero, J. Silvestre, Analysis of communication alternatives in a heterogeneous network for a supervision and control system, Computer Communications 29 (2006) 1133-1145.

[19] V. Casares-Giner, V. Pla, P. Escalle-Garcia, Mobility models for mobility management, in: D. Kouvatsos (Ed.), Network Performance Engineering, volume 5233 of Lecture Notes in Computer Science, 2011, Springer Berlin / Heidelberg, pp. 716-745. 
[20] A. Alshanyour, U. Baroudi, A simulation study: The impact of random and realistic mobility models on the performance of bypass-aodv in ad hoc wireless networks, EURASIP Journal on Wireless Communications and Networking 2010 (2010).

[21] M. Z. Aslam, A. R. Khan, Comparison of random waypoint \&amp; random walk mobility model under dsr, aodv \&amp; dsdv manet routing protocols, CoRR abs/1104.2368 (2011).

[22] T. Albero-Albero, Personal home page, formal models in detail, http://personales.alc.upv.es/vsempere/index_archivos/Page1082.htm.

Pdf thesis, http://riunet.upv.es/bitstream/handle/10251/10081/tesisUPV3452.pdf? sequence $=1$.

[23] T. Albero-Albero, S. Santonja-Climent, V.-M. Sempere-Payá, J. MataixOltra, Aodv performance evaluation and proposal of parameters modification for multimedia traffic on wireless ad hoc networks, in: Wireless Days (WD), 2009 2nd IFIP, Paris, France, pp. 1 -6. 